PAPER

\title{
Traumatic brain injury and grey matter concentration: a preliminary voxel based morphometry study
}

\author{
S D Gale, L Baxter, N Roundy, S C Johnson
}

J Neurol Neurosurg Psychiatry 2005;76:984-988. doi: 10.1136/jnnp.2004.036210

See end of article for authors' affiliations

.....

Correspondence to: Dr Shawn D Gale, Department of Clinical

Neuropsychology, Barrow

Neurological Institute, 222

W Thomas Rd, Suite 315 ,

Phoenix, Arizona 85013,

USA; s2gale@chw.edu

Received 9 January 2004

In revised form

1 September 2004

Accepted 11 October 2004
Background: Magnetic resonance imaging (MRI) studies have shown diffuse cerebral atrophy following traumatic brain injury. In the past, quantitative volumetric analysis of these changes was carried out by manually tracing specific regions of interest. In contrast, voxel based morphometry (VBM) is a fully automated technique that allows examination of the whole brain on a voxel by voxel basis.

Objective: To use VBM to evaluate changes in grey matter concentration following traumatic brain injury. Methods: Nine patients with a history of traumatic brain injury (ranging from mild to severe) about one year previously were compared with nine age and sex matched healthy volunteers. TI weighted three dimensional MRI images were acquired and then analysed with statistical parametric mapping software (SPM2). The patients with traumatic brain injury also completed cognitive testing to determine whether regional grey matter concentration correlated with a measure of attention and initial injury severity.

Results: Compared with controls, the brain injured patients had decreased grey matter concentration in multiple brain regions including frontal and temporal cortices, cingulate gyrus, subcortical grey matter, and the cerebellum. Decreased grey matter concentration correlated with lower scores on tests of attention and lower Glasgow coma scale scores.

Conclusions: Using VBM, regions of decreased grey matter concentration were observed in subjects with traumatic brain injury compared with well matched controls. In the brain injured patients, there was a relation between grey matter concentration and attentional ability.
A pproximately one million persons suffer a traumatic brain injury in the USA each year, with an estimated prevalence of 2.5 to 6.5 million. ${ }^{1}$ The mechanisms of neural injury ${ }^{2}$ as well as other factors associated with recovery of function have been well described. ${ }^{3}$ One of the most frequent complaints following traumatic brain injury is poor attention. ${ }^{14}$

Quantitative neuroimaging techniques, initially using computed tomography (CT) and then later using various magnetic resonance imaging (MRI) protocols, have produced evidence of both diffuse and focal changes in traumatic brain injury. ${ }^{5}$ Furthermore, markers of compromised cerebral integrity have been linked to neuropsychological and functional outcome. ${ }^{6-11}$ These quantitative techniques typically involved tracing regions of interest (ROI) identified by visual inspection. Thus, readily identifiable neuroanatomical structures such as the corpus callosum and hippocampus regions were defined a priori and then measured for change over time. ${ }^{11-15}$ However, regions that were less amenable to this technique received less consideration.

Voxel based morphometry (VBM) is an automated procedure carried out on MRI data acquired at high resolution in a three dimensional (whole brain) format, typically Tl spoiled gradient echo (SPGR). VBM is done using statistical parametric mapping (SPM) and has been described in great detail elsewhere ${ }^{16}$ Briefly, the images are normalised to stereotactic space, segmented into tissue type (grey matter, white matter, or cerebrospinal fluid) based on voxel intensity, and then smoothed. Statistical analyses can then be done to determine regional differences in grey matter concentration between groups. These analyses are based on the probability that any given voxel represents grey matter. Statistical parametric maps are then created using the general linear model ( see also Good et $a l^{17}$ ). VBM has been used in previous studies in a variety of populations including healthy participants, patients with various forms of dementia, and schizophrenic patients to identify regions of cerebral abnormality as well as to determine how these regions correlate with neuropsychological function. ${ }^{18-25}$

Our aim in this study was to identify regional differences in grey matter concentration between patients with a history of traumatic brain injury and controls. In addition, measures of attention and injury severity were correlated with grey matter concentration in brain injured patients to characterise the relation between cognitive function and regional grey matter concentration.

\section{METHODS \\ Subjects}

All subjects gave written informed consent in accordance with St Joseph's Hospital institutional review board.

Nine right handed patients with a history of traumatic brain injury (eight male, one female; mean (SD) age, 29.1 (7.8) years) were recruited as inpatients at St Joseph's Hospital and Medical Center in Phoenix, Arizona, as part of a larger study on recovery from brain injury. Exclusion criteria included a history of penetrating head injury or open skull fracture, previous neurosurgery, significant neurological disease unassociated with the injury, mental retardation, less than 10 years of formal education, severe psychiatric disease, and a history of alcohol or substance abuse within last six months. A medical history was collected, which including the post-resuscitation Glasgow coma scale (GCS) score at the time of admission to the hospital. GCS scores ranged from 5 to 15 (mean (SD), 9.1 (3.4); median 8.0). The patients returned approximately one year after their injury (mean 10.6 (2.4) months; median 11.0 months) for neurological, neuropsychological, and neuroimaging

Abbreviations: CPT-II, Conners continuous performance test, second edition; GCS, Glasgow coma scale; MNI, Montreal Neurological Institute; ROI, region of interest; SPGR, spoiled gradient echo; SPM, statistical parametric mapping; VBM, voxel based morphometry 
Table 1 Summary of demographic characteristics of the subject groups

\begin{tabular}{lrl}
\hline $\begin{array}{l}\text { Age } \\
\text { (years) }\end{array}$ & GCS & Initial CT findings \\
\hline 19 & 12 & $\begin{array}{l}\text { Right temporal haemorrhagic contusion } \\
41\end{array}$ \\
38 & 15 & Bilateral tentorial SDH, left>right \\
Left temporal epidural haematoma \\
23 & 7 & Right frontal hemorrhagic contusion \\
22 & 9 & Left frontal SAH, right tentorial SDH \\
26 & 7 & Bilateral temporal and right frontal contusions \\
36 & 6 & Right SAH, uncal herniation \\
31 & 5 & Epidural haematoma over right frontal lobe \\
26 & 13 & Left frontal SDH, bilateral frontal cortical contusion
\end{tabular}

$\mathrm{CT}$, computed tomography; GCS, Glasgow coma scale; $\mathrm{SAH}$ subarachnoid haematoma; SDH, subdural haematoma.

examinations. Demographic variables of the traumatic brain injury patients including initial CT findings are presented in table 1 .

Nine healthy right handed normal subjects were selected to create a control group with demographics closely matching the brain injured group. The controls were recruited from another study at our institution and consisted of eight men and one woman with a mean (SD) age of 28.8 (8.4) years. The controls were scanned on the same scanner with the same MRI protocols as the brain injured patients but did not undergo neuropsychological examination.

\section{MRI method}

Images were obtained on a $1.5 \mathrm{~T}$ GE Signa scanner (Milwaukee, Wisconsin, USA). High resolution Tl weighted images were acquired in the coronal plane with an SPGR gradient echo pulse sequence. Imaging parameters were: TR (time of repetition $)=24 ;$ TE $($ time of echo $)=6$; flip

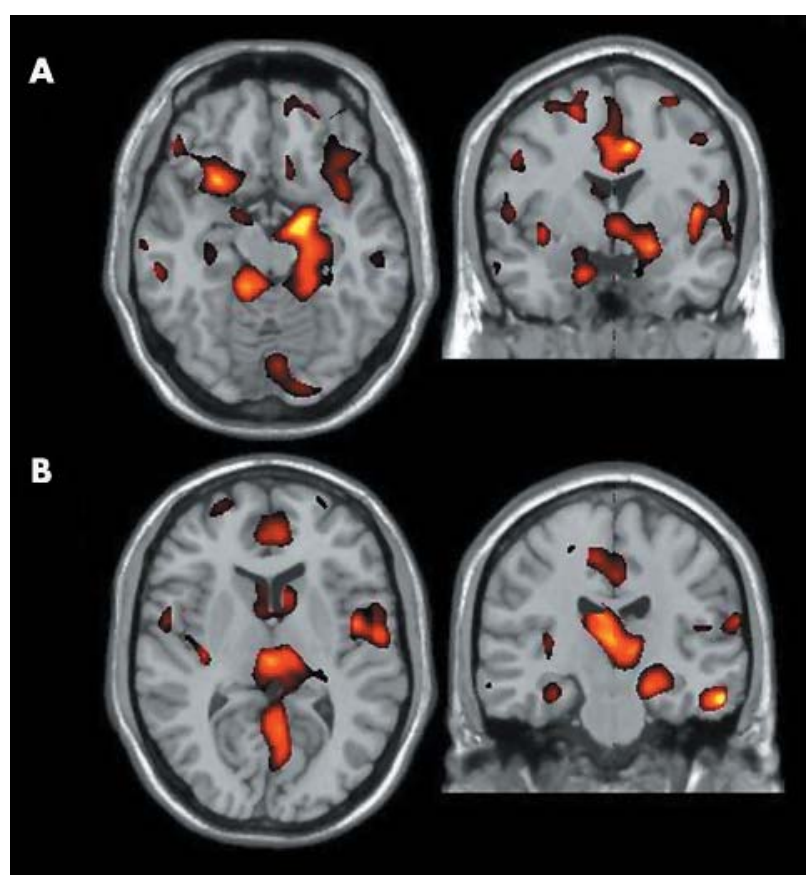

Figure 1 Regions of decreased grey matter concentration $(p<0.005)$ in the traumatic brain injury group compared with the normal controls superimposed on a normal brain template. Images are in neurological orientation (right on right). Panel A shows a large cluster of contiguous voxels in the right mesial temporal region including the

parahippocampal gyrus and extending into the right cerebral peduncle. Panel $B$ demonstrates how this cluster extends upwards into the right and left thalamus and caudate. angle $=40$; NEX (number of excitations) $=1$; slice thickness $=1.5 \mathrm{~mm}$; 0 gap between slices; field of view $=24 \mathrm{~cm}$; in plane resolution $=0.9375 \mathrm{~mm}^{2}$ voxels.

\section{Image analysis}

The optimised VBM procedure was carried out according to the protocol of Good et al. ${ }^{17}$ Acquired images were processed and analysed with the statistical parametric mapping software SPM2 (Wellcome Department of Cognitive Neurology, London, UK). Segmented grey matter images were spatially smoothed with a $12 \mathrm{~mm}$ full width at half maximum isotropic Gaussian kernel. The smoothed grey matter images were normalised to a global mean pixel value of 100 .

\section{Statistical analysis}

SPM results comparing group differences in grey matter concentration were thresholded to a probability of $\mathrm{p}=0.005$ (uncorrected) with the extent threshold set at 250 voxels $\left(0.25 \mathrm{~cm}^{3}\right)$ and local maxima more than $8.0 \mathrm{~mm}$ apart. SPM results correlating grey matter concentration with cognitive performance and injury severity were thresholded to $p \leqslant 0.01$ (uncorrected) because of the exploratory nature of this study and the small sample size. Only clusters of at least 250 voxels were included in the analysis. Local maxima more than $8.0 \mathrm{~mm}$ apart are reported, including cluster size and $\mathrm{T}$ value for each cluster. Locations for statistical findings are reported in the standard coordinate space $(X, Y, Z)$ developed by the International Consortium for Brain Mapping, commonly referred to as the MNI (Montreal Neurological Institute) atlas. The correlation between GCS and grey matter concentration was determined using the Spearman rank correlation coefficient $\left(r_{\mathrm{s}}\right)$.

\section{Neuropsychological measures}

The second edition of Conners' continuous performance test $(\mathrm{CPT}-\mathrm{II})^{26}$ was used to measure attention. This task requires the subject to respond, by button pressing, to all stimuli (that is, letters) except a specified target item (the letter " $\mathrm{x}$ "). The variable of interest was $d$ prime $\left(\mathrm{d}^{\prime}\right)$, a measure of attentiveness based on the ability to discriminate target from non-target items. ${ }^{26}$ Results from the CPT-II are reported as T scores which have a mean of 50 and standard deviation of 10. On the CPT-II, higher T scores represent greater impairment. However, because $\mathrm{T}$ scores in clinical neuropsychology are usually reported with lower scores representing greater cognitive impairment, the $\mathrm{T}$ scores from the CPT-II were linearly transformed to conform to this practice. The CPT-II was administered on the day the MRI was obtained.

This study used a general measure of injury severity, the Glasgow coma scale (GCS), ${ }^{27}$ obtained at the time of initial hospital admission. While this measure has been shown to be affected by a variety of factors, it has been associated with functional outcome, ${ }^{28}$ quantitative volumetric analyses, ${ }^{6}$ and cognitive performance ${ }^{11}$ following traumatic brain injury.

\section{RESULTS \\ Group comparison}

Group comparisons between the brain injured and control subjects showed diffuse regional decreases in grey matter concentration in the brain injury group. Specifically, decreased grey matter concentration was found in bilateral frontal, temporal, and parietal lobes as well as in the cerebellum. Decreased grey matter concentration was generally more predominant in the right hemisphere. A large cluster of decreased grey matter concentration encompassing the right mesial temporal region including the parahippocampal gyrus and extending into the right cerebral peduncle is depicted in panel A of fig 1. The cluster extends upwards into the right and left thalamus and caudate nucleus (panel B). A listing of anatomical regions, MNI atlas coordinates $(\mathrm{X}, \mathrm{Y}, \mathrm{Z})$, cluster sizes, 
Table 2 Regions of significant atrophy in nine patients with traumatic brain injury compared with nine healthy controls

\begin{tabular}{llrl}
\hline Anatomical region & $\mathbf{X}, \mathbf{Y}, \mathbf{Z}^{*}$ & Cluster & $\mathrm{T} \dagger$ \\
\hline Right frontal & $30,-5,64$ & 634 & 5.08 \\
Right temporal & $57,-19,-22$ & 1051 & 4.97 \\
Right temporal/parahippocampal/hippocampus/ & $14,-8,-15$ & 9324 & 4.86 \\
cerebral peduncle/thalamus/caudate & $18,-90,-23$ & 1509 & 4.65 \\
Right cerebellum & $7,2,40$ & 8106 & 4.50 \\
Right cingulate & $0,-50,24$ & 5443 & 4.32 \\
Right posterior cingulate/left parietal precuneus & $-48,9,26$ & 359 & 4.26 \\
Left frontal & $39,19,41$ & 396 & 4.26 \\
Right frontal & $-14,-42,-11$ & 1753 & 4.24 \\
Left cerebellum & $-30,17,-15$ & 873 & 4.15 \\
Left frontal & $4,14,-7$ & 2097 & 4.12 \\
Right anterior cingulate & $43,0,5$ & 2015 & 4.03 \\
Right insula & $56,-9,9$ & 325 & 3.88 \\
Right temporal & $1,-39,59$ & 539 & 3.87 \\
Right parietal & $-1,-76,-43$ & 617 & 3.87 \\
Left cerebellum & $51,-51,-27$ & 316 & 3.35 \\
Right cerebellum & & & \\
\hline *Coordinates at the peak voxel in each cluster based on the Montreal Neurological Institute atlas. & \\
tp $\leqslant 0.001$. & & &
\end{tabular}

and T values is provided in table 2. SPM2 results showing areas of decreased grey matter concentration in the brain injured group compared with the normal controls are superimposed on a normal brain template in fig 1 .

\section{Neuropsychological measures and grey matter concentration in brain injured patients}

We undertook an analysis of the relation between grey matter concentration and attention among the brain injured patients. Performance on the CPT-II was positively correlated

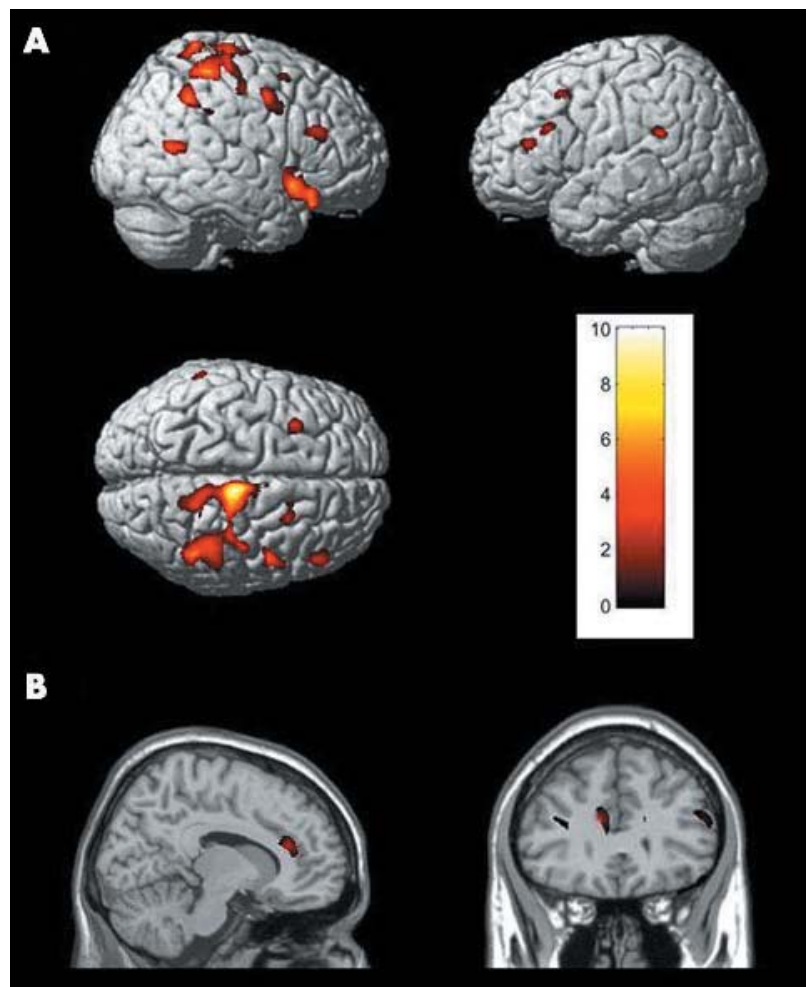

Figure 2 Simple regression (positive) of CPT-II $d^{\prime}$ and grey matter concentration in patients with traumatic brain injury superimposed on a normal brain template. Images are in neurological orientation (right on right). Panel A shows several right hemisphere regions, mainly frontal and parietal. Panel B illustrates an association between performance on the CPT-II and grey matter concentration in the left cingulate and frontal lobe. with grey matter concentration. Specifically, decreased performance was associated with decreased grey matter concentration in several right hemisphere regions-mainly frontal, temporal, and parietal-as shown in fig 2, panel A. In addition, poorer performance on the CPT-II also correlated with decreased grey matter concentration in the left cingulate and frontal lobe (panel B). The results are summarised in more detail in table 3 . The clusters of contiguous voxels are reported in order of decreasing statistical significance.

Glasgow coma scale score correlated with multiple regions and a particularly large cluster had the most significant maxima in the left insular cortex (MNI coordinates - 37, 3, 7) but also included bilateral caudate, putamen, and thalamus. In addition, right parietal and occipital and left frontal, temporal, and occipital regions of decreased grey matter concentration were found and are reported in table 3. Results from simple regression analyses between grey matter concentration and GCS superimposed on a normal brain template are presented in fig 3 . Figure 4 shows the relation between grey matter concentration from the cluster with a peak value at MNI coordinates $-37,3,7$ and the score on the GCS on hospital admission in the brain injured patients. The Spearman rank-correlation coefficient $\left(r_{\mathrm{s}}\right)$ depicted in this figure is $0.803(\mathrm{p}=0.009)$.

\section{DISCUSSION}

This study showed that diffuse changes in cerebral grey matter can be observed following traumatic brain injury. These changes were found postacutely, approximately one year after the injury. The significant decrease in grey matter concentration in the brain injured patients compared with age and sex matched controls was consistent with previous quantitative neuroimaging studies demonstrating postinjury changes. Both the current study and others show that changes in cerebral integrity related to traumatic brain injury occur in a variety of neuroanatomical regions including the frontal and temporal lobes, the limbic system, and the subcortical regions. ${ }^{5}$ Furthermore, this study emphasises that common areas of residual injury across individual patients can be observed despite differences in injury severity and acute neuroimaging findings.

Previous studies have suggested that white matter structures are more likely to be affected following traumatic brain injury than grey matter structures. ${ }^{511} 2930$ However, diffuse cerebral atrophy is also a common finding after traumatic brain injury in the postacute phase, suggesting that grey and white matter changes are not mutually exclusive. ${ }^{5611}$ For 
Table 3 Regions of grey matter concentration correlated (positive regression) with attention and initial injury severity in traumatic brain injury patients

\begin{tabular}{|c|c|c|c|c|}
\hline Neuropsychological test & Anatomical structure & $\mathrm{X}, \mathrm{Y}, \mathrm{Z}^{*}$ & Cluster & T† \\
\hline \multirow[t]{12}{*}{ CPT-II d' } & Right frontal & $17,37,25$ & 393 & 22.57 \\
\hline & Left anterior cingulate & $-13,31,19$ & 771 & 10.01 \\
\hline & Right parietal & $48,-48,41$ & 1038 & 9.11 \\
\hline & Left frontal & $-32,16,46$ & 366 & 8.26 \\
\hline & Right frontal & $7,-18,70$ & 10797 & 7.59 \\
\hline & Right frontal & $51,26,21$ & 486 & 6.63 \\
\hline & Right temporal & $51,16,-12$ & 3013 & 6.57 \\
\hline & Right temporal & $47,-61,13$ & 872 & 6.40 \\
\hline & Right parietal & $16,-50,35$ & 329 & 5.88 \\
\hline & Left frontal & $-39,26,25$ & 261 & 5.66 \\
\hline & Right frontal & $14,50,0$ & 449 & 5.25 \\
\hline & Right frontal & $49,1,43$ & 766 & 5.17 \\
\hline \multirow[t]{11}{*}{ GCS } & Right parietal & $1,-54,54$ & 1763 & 9.01 \\
\hline & Left temporal & $-62,-12,0$ & 1073 & 8.07 \\
\hline & $\begin{array}{l}\text { Left insula, bilateral caudate, } \\
\text { putamen, thalamus }\end{array}$ & $-37,3,7$ & 13676 & 7.02 \\
\hline & Left frontal & $-42,21,53$ & 2521 & 6.97 \\
\hline & Right occipital & $8,-99,14$ & 4882 & 6.51 \\
\hline & Left frontal & $-39,34,3$ & 297 & 5.71 \\
\hline & Right occipital/temporal/ & & & \\
\hline & left occipital & $42,-68,6$ & 16482 & 5.54 \\
\hline & Left frontal & $-21,19,61$ & 502 & 5.27 \\
\hline & Right insula & $39,1,-1$ & 711 & 5.09 \\
\hline & Left posterior cingulate & $-10,-22,45$ & 434 & 5.04 \\
\hline
\end{tabular}

example, Anderson and Bigler ${ }^{29}$ analysed day of injury and postacute neuroimaging in a group of patients with traumatic brain injury and found that dilatation of the anterior horns of the lateral ventricles was associated with a decrease in the corpus callosum, although the volume of the caudate nucleus remained unchanged. However, the analysis was restricted to the region of the caudate and nearby structures, so these findings may not generalise to other regions. That study also used measurements based on a single axial slice rather than a

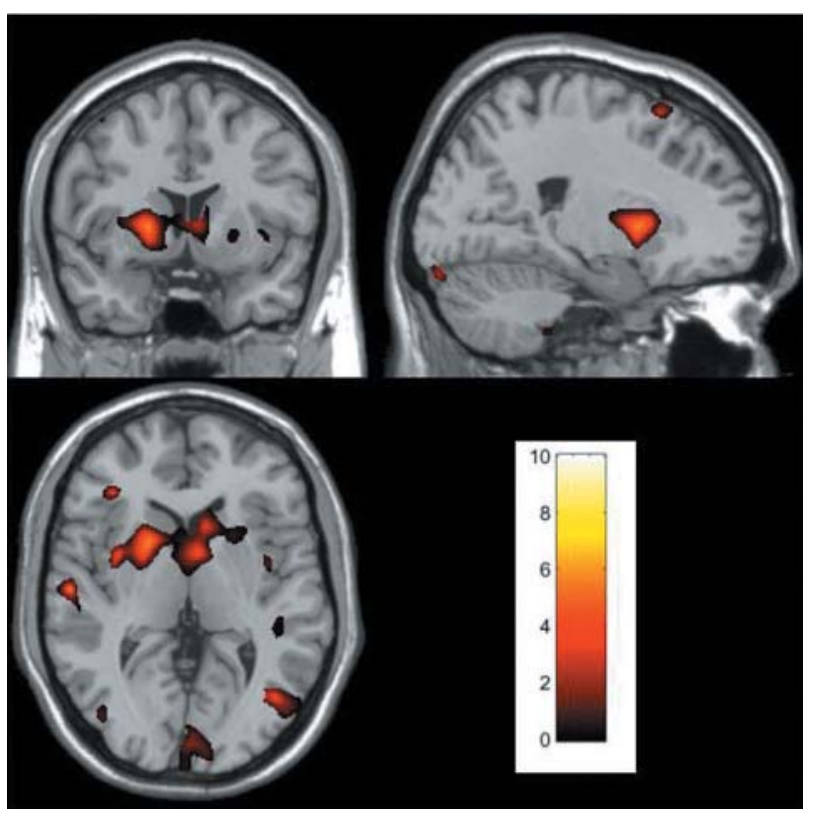

Figure 3 Simple regression (positive) of admission Glasgow coma scale score and grey matter concentration in patients with traumatic brain injury superimposed on a normal brain template. Images are in neurological orientation (right on right). This large cluster of subcortical voxels includes the left insular cortex and bilateral caudate, putamen, and thalamus. whole brain analysis. Furthermore, it has been suggested that longitudinal investigations of cerebral changes are time dependent. ${ }^{56}$ Thus changes specific to either grey or white matter may also be time related, requiring a longitudinal analysis of brain changes. Whole brain analyses including both grey and white matter over time are currently under way in our laboratory.

It has been suggested that loss of consciousness and coma may represent a disconnection between cortical centres as well as between cortical and subcortical regions. ${ }^{31}$ Deep lesions (for example, in the thalamus and midbrain) have been associated with lower GCS scores ${ }^{32}$ as well as worse functional outcomes. ${ }^{33}{ }^{34}$ Consistent with those studies, we found decreased grey matter concentration in various subcortical regions including the caudate, putamen, thalamus, and even the cerebellum. Similarly, decreased grey matter concentration in cortical regions functionally connected to these subcortical regions was also shown. Figure 4 illustrates the relation between grey matter concentration from a large cluster with a peak value at MNI coordinates $-37,3,7$ and the score on the Glasgow coma scale on hospital admission in the patients with traumatic brain injury. The strong correlation depicted in this figure suggests

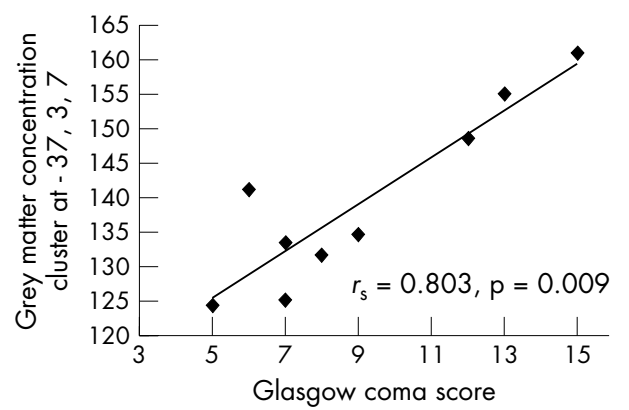

Figure 4 Spearman rank correlation between admission Glasgow coma scale score and grey matter concentration voxel cluster at Montreal Neurological Institute coordinates $-37,3,7$ (see fig 3). 
that greater injury severity was associated with a greater decrease in grey matter concentration. However, this finding must be interpreted with caution as it is possible that it could be an artefact of the small sample size.

A strong correlation between grey matter concentration and attention (CPT-II) was also observed in the frontal and temporal regions in the brain injured patients. Impairment of attention following traumatic brain injury has repeatedly been demonstrated (for a review, see Cossa and Fabiani ${ }^{35}$ ). Previous studies using the CPT have reported impairment in sustained attention among patients with right frontal lesions, ${ }^{36}$ reduced metabolism in medial and inferior frontal cortex on PET scans in patients with schizophrenia, ${ }^{37}$ and correlations between the CPT and decreased grey matter concentration in the thalamus, temporal lobe, and frontal lobe in patients with schizophrenia. ${ }^{19}$ McAllister et $a l^{38}$ studied patients with mild traumatic brain injury at one month after their injury by functional magnetic resonance imaging (fMRI), using a working memory task, and found that when the attentional demand was low the brain injured patients activated in a similar pattern to that of the controls in the bilateral posterior parietal and dorsolateral prefrontal cortex. However, when the working memory load increased, the brain injured patients showed greater activation in the right dorsolateral prefrontal cortex and posterior parietal regions than the controls. This pattern is consistent with the theory that the right hemisphere may play a preferential role in attentional processes. ${ }^{39}$ In our study, decreased attention was also correlated preferentially with decreased grey matter concentration in the right hemisphere, providing further support for the view that the integrity of the right hemisphere is critical in attentional control.

Owing to the exploratory and descriptive nature of this study, our statistical analysis was not stringent; nevertheless, our findings were generally consistent with findings from previous neuroimaging and neuropsychological studies in patients with traumatic brain injury. ${ }^{5611} 323435$ Our study shows that within a small sample of patients with traumatic brain injury it is possible to detect common regions that are vulnerable to trauma. However, this preliminary investigation is limited in its generalisability because of the small sample size. Further studies with larger numbers and collaborating ROI analyses are needed to clarify the course of grey matter changes after traumatic brain injury. To this end, VBM may be a promising tool in assessing the cerebral changes that influence recovery, as well as in generating hypotheses for future outcomes research, because it allows whole brain, voxel by voxel, analysis of grey matter concentration in a non-biased fashion.

\section{ACKNOWLEDGEMENT}

Supported by NIH grant MH65723.

\footnotetext{
Authors' affiliations

S D Gale, L Baxter, N Roundy, Barrow Neurological Institute, Phoenix, Arizona, USA

S C Johnson, University of Wisconsin Medical School, Madison, Wisconsin, USA

Competing interests: none declared

\section{REFERENCES}

1 NIH Consensus Statement. Rehabilitation of persons with traumatic brain injury. NIH Consensus Statement 1998 October 26-28;16(1):1-41.

2 Povlishock JT, Christman CW. The pathobiology of traumatically induced axonal injury in animals and humans: a review of current thoughts. J Neurotrauma 1995; 12:555-64.

3 Prigatano GP. Principles of neuropsychological rehabilitation. New York: Oxford University Press, 1999.

4 van Zomeren $\mathrm{AH}$, van den Burg W. Residual complaints of patients two years after severe head injury. J Neurol Neurosurg Psychiatry 1985;48:21-8.

5 Bigler, ed. Quantitative magnetic resonance imaging in traumatic brain injury. J Head Trauma Rehabil 2001;16:117-34.
}

6 Blatter DD, Bigler ED, Gale SD, et al. MR-based brain and cerebrospinal fluid measurement after traumatic brain injury: correlation with neuropsychological outcome. Am J Neuroradiol 1997; 18:1-10

7 Bigler ED, Blatter DD, Anderson CV, et al. Hippocampal volume in normal aging and traumatic brain injury. Am J Neuroradiol 1997;18:11-23.

8 Henry-Feugeas MC, Azouvi P, Fontaine A, et al. MRI analysis of brain atrophy after severe closed-head injury: relation to clinical status. Brain Inj 2000; 14:597-604.

9 van der Naalt J, van Zomeren AH, Sluiter WJ, et al. One year outcome in mild to moderate head injury: the predictive value of acute injury characteristics related to complaints and return to work. I Neurol Neurosurg Psychiatry 1999;66:207-13.

10 Pierallini A, Pantano P, Fantozzi LM, et al. Correlation between MRI findings and long-term outcome in patients with severe brain trauma. Neuroradiology 2000;42:860-7.

11 Gale SD, Johnson SC, Bigler ED, et al. Nonspecific white matter degeneration following traumatic brain injury. J Int Neuropsychol Soc 1995; 1:17-28.

12 Levin HS, Williams DH, Valastro M, et al. Corpus callosal atrophy following closed head injury: detection with magnetic resonance imaging. J Neurosurg 1990;73:77-81.

13 Johnson SC, Farnworth T, Pinkston JB, et al. Corpus callosum surface area across the human adult life span: effect of age and gender. Brain Res Bull 1994:35:373-7.

14 Gale SD, Burr RB, Bigler ED, et al. Fornix degeneration and memory in traumatic brain injury. Brain Res Bull 1993;32:345-9.

15 Tate DF, Bigler, eds. Fornix and hippocampal atrophy in traumatic brain injury. Learn Mem 2000;7:442-6.

16 Ashburner J, Friston KJ. Voxel-based morphometry - the methods. Neuroimage 2000;11:805-21.

17 Good CD, Johnsrude IS, Ashburner J, et al. A voxel-based morphometric study of ageing in 465 normal adult human brains. Neuroimage 2001;14:21-36.

18 Boxer AL, Rankin KP, Miller BL, et al. Cinguloparietal atrophy distinguishes Alzheimer disease from semantic dementia. Arch Neurol 2003;60:949-56.

19 Salgado-Pineda P, Baeza I, Perez-Gomez M, et al. Sustained attention impairment correlates to gray matter decreases in first episode neurolepticnaive schizophrenic patients. Neuroimage 2003;19:365-75.

20 Karas GB, Burton EJ, Rombouts SA, et al. A comprehensive study of gray matter loss in patients with Alzheimer's disease using optimized voxel-based morphometry. Neuroimage 2003; 18:895-907

21 Baron JC, Chetelat G, Desgranges B, et al. In vivo mapping of gray matter loss with voxel-based morphometry in mild Alzheimer's disease. Neuroimage 2001;14:298-309.

22 Rosen HJ, Gorno-Tempini ML, Goldman WP, et al. Patterns of brain atrophy in frontotemporal dementia and semantic dementia. Neurology 2002; 58: 198-208.

23 Wilke M, Kaufmann C, Grabner A, et al. Gray matter-changes and correlates of disease severity in schizophrenia: a statistical parametric mapping study. Neuroimage $2001 ; 13: 814-24$

24 Maguire EA, Gadian DG, Johnsrude IS, et al. Navigation-related structural change in the hippocampi of taxi drivers. Proc Natl Acad Sci USA 2000:97:4398-403.

25 Wright IC, McGuire PK, Poline JB, et al. A voxel-based method for the statistical analysis of gray and white matter density applied to schizophrenia. Neuroimage 1995;2:244-52.

26 Conners CK. Conners' Continuous Performance Test-II. Toronto: Multi-Health Systems Inc, 2000.

27 Teasdale G, Jennett B. Assessment of coma and impaired consciousness. A practical scale. Lancet 1974;ii:81-4.

28 Dikmen SS, Machamer JE, Winn HR, et al. Neuropsychological outcome at 1year post head injury. Neuropsychology 1995;9:80-90.

29 Anderson CV, Bigler, eds. The role of caudate nucleus and corpus callosum atrophy in trauma-induced anterior horn dilation. Brain Inj 1994;8:565-9.

30 Gale SD, Johnson SC, Bigler ED, et al. Trauma-induced degenerative changes in brain injury: a morphometric analysis of three patients with preinjury and postinjury MR scans. J Neurotrauma 1995:12:151-8.

31 Povlishock JT, Kontos HA, Ellis EF. Current thoughts on experimental head injury. In: Becker DP, Gudeman SK, eds. Textbook of head injury. Philadelphia: WB Saunders, 1989:451-65.

32 Prat R, Calatayud-Maldonado V. Prognostic factors in postraumatic severe diffuse brain injury. Acta Neurochir (Wien) 1998;140:1257-60; discussion 1261

33 Levin HS, Mendelsohn D, Lilly MA, et al. Magnetic resonance imaging in relation to functional outcome of pediatric closed head injury: a test of the OmmayaGennarelli model. Neurosurgery 1997:40:432-40, discussion 440-1.

34 Levin HS, Williams D, Crofford MJ, et al. Relationship of depth of brain lesions to consciousness and outcome after closed head injury. J Neurosurg 1988;69:861-6.

35 Cossa FM, Fabiani M. Attention in closed head injury: a critical review. Ital J Neurol Sci 1999;20:145-53.

36 Rueckert L, Grafman J. Sustained attention deficits in patients with right frontal lesions. Neuropsychologia 1996;34:953-63.

37 Buchsbaum MS, Haier RJ, Potkin SG, et al. Frontostriatal disorder of cerebral metabolism in never-medicated schizophrenics. Arch Gen Psychiatry 1992;49:935-42.

38 McAllister TW, Saykin AJ, Flashman LA, et al. Brain activation during working memory 1 month after mild traumatic brain injury: a functional MRI study. Neurology 1999:53:1300-8.

39 Mesulam MM. Attentional networks, confusional states, and neglect syndromes. In: Principles of behavioral and cognitive neurology. Oxford University Press: New York, 2000:1-173. 